\title{
2-D Deformation analysis of a half-space due to a long dip-slip fault at finite depth
}

\author{
Sushil K TOMAR ${ }^{1}$ and NAREsh K Dhiman ${ }^{2}$ \\ ${ }^{1}$ Department of Mathematics, Panjab University, Chandigarh 160 014, India. \\ e-mail:sktomar@yahoo.com \\ ${ }^{2}$ S. U. S. College of Engineering and Technology, Tangori (Mohali) 140 306, Punjab, India.
}

Closed form analytical expressions of stresses and displacements at any field point due to a very long dip-slip fault of finite width buried in a homogeneous, isotropic elastic half-space, are presented. Airy stress function is used to derive the expressions of stresses and displacements which depend on the dip angle and depth of the upper edge of the fault. The effect of dip angle and depth of the upper edge of the fault on stresses and displacements is studied numerically and the results obtained are presented graphically. Contour maps for stresses and displacements are also presented. The results of Rani and Singh (1992b) and Freund and Barnett (1976) have been reproduced.

\section{Introduction}

The problems relating to the application of the elastic dislocation theory to earth faulting have been investigated by many researchers and have appeared in the open literature. Savage (1974) among others is an important reference in the area of dislocation theory. 2-D dip-slip dislocation models of faulting have been used extensively to model the lithospheric deformation associated with faulting; see, Cohen (1992) and the references listed therein. Freund and Barnett (1976) developed a 2-D model of dip-slip faulting in a uniform halfspace and obtained the relationship between fault slip and surface deformation. The problem of static deformation in a uniform half-space due to long faults has been attempted by a number of researchers. Singh and Rani (1996) presented stepby-step progress made in the direction of crustal deformation modeling associated with strike-slip and dip-slip faulting in the earth. Cohen (1996) gave convenient formulas for determining dip-slip parameters for geophysical observables. Singh et al (1997) investigated the problem of deformation of a layered half-space due to a very long dip-slip fault. Okada $(1985,1992)$ investigated 3-D prob- lems of surface deformation and internal deformation due to shear and tensile faults in a halfspace. Rani and Singh (1992b) attempted the static problem of elastic deformation of a half-space due to a long dip-slip fault of finite width. They obtained closed form expressions of the displacements and stresses at an arbitrary point of a uniform half-space. Other notable references in this field of study are Maruyama (1964, 1966); Savage (1980); Rybicki (1986); Rani et al (1991); Rani and Singh (1992a); Singh et al (1992); Singh and Rani (1993); and Singh et al (1994). Recently, Singh and Singh (2000) and Singh et al (2002) studied similar problems due to tensile fault.

In the present analysis, a 2-D faulting problem is investigated. We have obtained the expressions for displacements and stresses at an arbitrary field point of a uniform half-space due to a long dip-slip inclined fault. It is believed that all the faults are not surface breaking and blind faults are also there beneath the ground surface, therefore, we have considered the upper edge of the inclined dip-slip fault at some finite depth $h$ below the earth surface. By adopting simple and straightforward techniques we have been able to obtain the analytical results for 2-D case, which have not been derived by Okada

Keywords. Deformation; Airy stress function; dip-slip fault; contour map.

Proc. Indian Acad. Sci. (Earth Planet. Sci.), 112, No. 4, December 2003, pp. 587-596

(C) Printed in India. 
(1992) and do not form the special case derived from the results obtained by him.

\section{Stresses and displacements}

We consider a long dip-slip inclined fault, whose upper edge is at a depth $h$ below the earth surface. We take the origin, $x$ and $y$-axis on the surface of the earth and $z$-axis pointing downward (see figure 1). We shall use the notations $x=$ $x_{1}, y=x_{2}, z=x_{3}$. The problem is 2-dimensional in $x_{2}-x_{3}$ plane, in which the displacement components $u_{i}$ in the direction of $x_{i}(i=1,2,3)$ are independent of $x_{1}$ co-ordinate so that $u_{1}=0$ and $\partial / \partial x_{1} \equiv 0$. As given in Rani and Singh (1992b), the Airy stress function $U$ for dip-slip line source locating at $\left(y_{2}, y_{3}\right)$ is given by

$$
\begin{aligned}
U= & \frac{\mu b d s}{2 \pi(1-\sigma)} \\
& \left\{\operatorname { c o s } 2 \delta \left[\left(x_{2}-y_{2}\right)\left(x_{3}-y_{3}\right)\left(\frac{1}{R_{1}^{2}}-\frac{1}{R_{2}^{2}}\right)\right.\right. \\
& \left.+4 x_{3} y_{3}\left(x_{2}-y_{2}\right)\left(x_{3}+y_{3}\right) \frac{1}{R_{2}^{4}}\right] \\
& +\sin 2 \delta\left[\left(x_{3}-y_{3}\right)^{2} \frac{1}{R_{1}^{2}}+\left(x_{3}^{2}-y_{3}^{2}+2 x_{3} y_{3}\right) \frac{1}{R_{2}^{2}}\right. \\
& \left.\left.-4 x_{3} y_{3}\left(x_{3}+y_{3}\right)^{2} \frac{1}{R_{2}^{4}}\right]\right\}
\end{aligned}
$$

where

$$
\begin{aligned}
& R_{1}^{2}=\left(x_{2}-y_{2}\right)^{2}+\left(x_{3}-y_{3}\right)^{2}, \\
& R_{2}^{2}=\left(x_{2}-y_{2}\right)^{2}+\left(x_{3}+y_{3}\right)^{2} .
\end{aligned}
$$

Substituting $y_{2}=s \cos \delta$ and $y_{3}=h+s \sin \delta$ into equation (1) and integrating over $s$ between the limits 0 to $L$, where $L=s_{2}-s_{1}\left(s_{1} \leq s \leq s_{2}\right)$ is the width of the fault, we obtain

$$
\begin{aligned}
U= & \frac{\mu b}{2 \pi(1-\sigma)}\left[\left(x_{2} \sin \delta-x_{3} \cos \delta+h \cos \delta\right) \ln \frac{R_{1}}{R_{2}}\right. \\
& +2 x_{3}\left\{\sin \delta\left(x_{2} \sin \delta+x_{3} \cos \delta\right) s\right. \\
& \left.\left.+h\left[\left(x_{3}+h\right) \cos \delta+\left(x_{2}+s \cos \delta\right) \sin \delta\right]\right\} \frac{1}{R_{2}^{2}}\right]\left.\right|_{0} ^{L},
\end{aligned}
$$

and the above expressions of $R_{1}^{2}$ and $R_{2}^{2}$ now take the following form

$$
\begin{aligned}
& R_{1}^{2}=\left(x_{2}-s \cos \delta\right)^{2}+\left(x_{3}-h-s \sin \delta\right)^{2}, \\
& R_{2}^{2}=\left(x_{2}-s \cos \delta\right)^{2}+\left(x_{3}+h+s \sin \delta\right)^{2} .
\end{aligned}
$$

Using the well known relations between the Airy stress function $U$ and the stresses $p_{i j}$, we obtain $p_{22}=\frac{\mu b}{2 \pi(1-\sigma)}\left\{\left(x_{2} \sin \delta-3 x_{3} \cos \delta+h \cos \delta\right)\right.$

$$
\begin{aligned}
& \left(\frac{1}{R_{1}^{2}}-\frac{1}{R_{2}^{2}}\right)+(s \sin 2 \delta+2 h \cos \delta)\left(\frac{1}{R_{1}^{2}}+\frac{3}{R_{2}^{2}}\right) \\
& -2\left(x_{2} \sin \delta-x_{3} \cos \delta+h \cos \delta\right)
\end{aligned}
$$$$
\left[\left(x_{3}-h-s \sin \delta\right)^{2} \frac{1}{R_{1}^{4}}-\left(x_{3}+h+s \sin \delta\right)^{2} \frac{1}{R_{2}^{4}}\right]
$$

$-4\left[s \sin \delta\left(3 x_{2} x_{3} \sin \delta+5 x_{3}^{2} \cos \delta\right.\right.$

$\left.+2 s \sin \delta\left[2 x_{3} \cos \delta+x_{2} \sin \delta\right]\right)$

$+2 h\left(x_{3}+h+s \sin \delta\right)\left[\left(2 x_{3}+h\right) \cos \delta\right.$

$\left.+\left(x_{2}+s \cos \delta\right) \sin \delta\right]$

$+2 h\left(x_{2} \sin \delta+2 x_{3} \cos \delta\right) s \sin \delta$

$\left.+h x_{3}\left[\left(x_{3}+h\right) \cos \delta+\left(x_{2}+s \cos \delta\right) \sin \delta\right]\right] \frac{1}{R_{2}^{4}}$

$+16\left(x_{3}+h+s \sin \delta\right)^{2} x_{3}\left[\sin \delta\left(x_{2} \sin \delta+x_{3} \cos \delta\right) s\right.$

$$
\left.\left.+h\left\{\left(x_{3}+h\right) \cos \delta+\left(x_{2}+s \cos \delta\right) \sin \delta\right\}\right] \frac{1}{R_{2}^{6}}\right\}\left.\right|_{0} ^{L},
$$

$$
\begin{aligned}
& p_{23}=\frac{\mu b}{2 \pi(1-\sigma)}\left\{\left(x_{2} \cos \delta-x_{3} \sin \delta+h \sin \delta\right)\right. \\
& \left(\frac{1}{R_{1}^{2}}-\frac{1}{R_{2}^{2}}\right)+s \cos 2 \delta\left(\frac{1}{R_{1}^{2}}-\frac{1}{R_{2}^{2}}\right) \\
& +2\left(x_{2} \sin \delta-x_{3} \cos \delta+h \cos \delta\right)\left(x_{2}-s \cos \delta\right) \\
& \quad\left[\left(x_{3}-h-s \sin \delta\right) \frac{1}{R_{1}^{4}}-\left(x_{3}+h+s \sin \delta\right) \frac{1}{R_{2}^{4}}\right] \\
& +4\left(x_{2}-s \cos \delta\right)\left[s \sin \delta\left(x_{2} \sin \delta+2 x_{3} \cos \delta\right)\right. \\
& \left.+h\left\{\left(2 x_{3}+h\right) \cos \delta+\left(x_{2}+s \cos \delta\right) \sin \delta\right\}\right] \frac{1}{R_{2}^{4}} \\
& +4\left(x_{3}+h+s \sin \delta\right) x_{3}\left(s \sin ^{2} \delta+h \sin \delta\right) \frac{1}{R_{2}^{4}} \\
& -16\left(x_{2}-s \cos \delta\right)\left(x_{3}+h+s \sin \delta\right) \\
& x_{3}\left\{s \sin \delta\left(x_{2} \sin \delta+x_{3} \cos \delta\right)+h\left[\left(x_{3}+h\right) \cos \delta\right.\right. \\
& \left.\left.\left.+\left(x_{2}+s \cos \delta\right) \sin \delta\right]\right\} \frac{1}{R_{2}^{6}}\right\}\left.\right|_{0} ^{L},
\end{aligned}
$$


$\mathrm{O}$

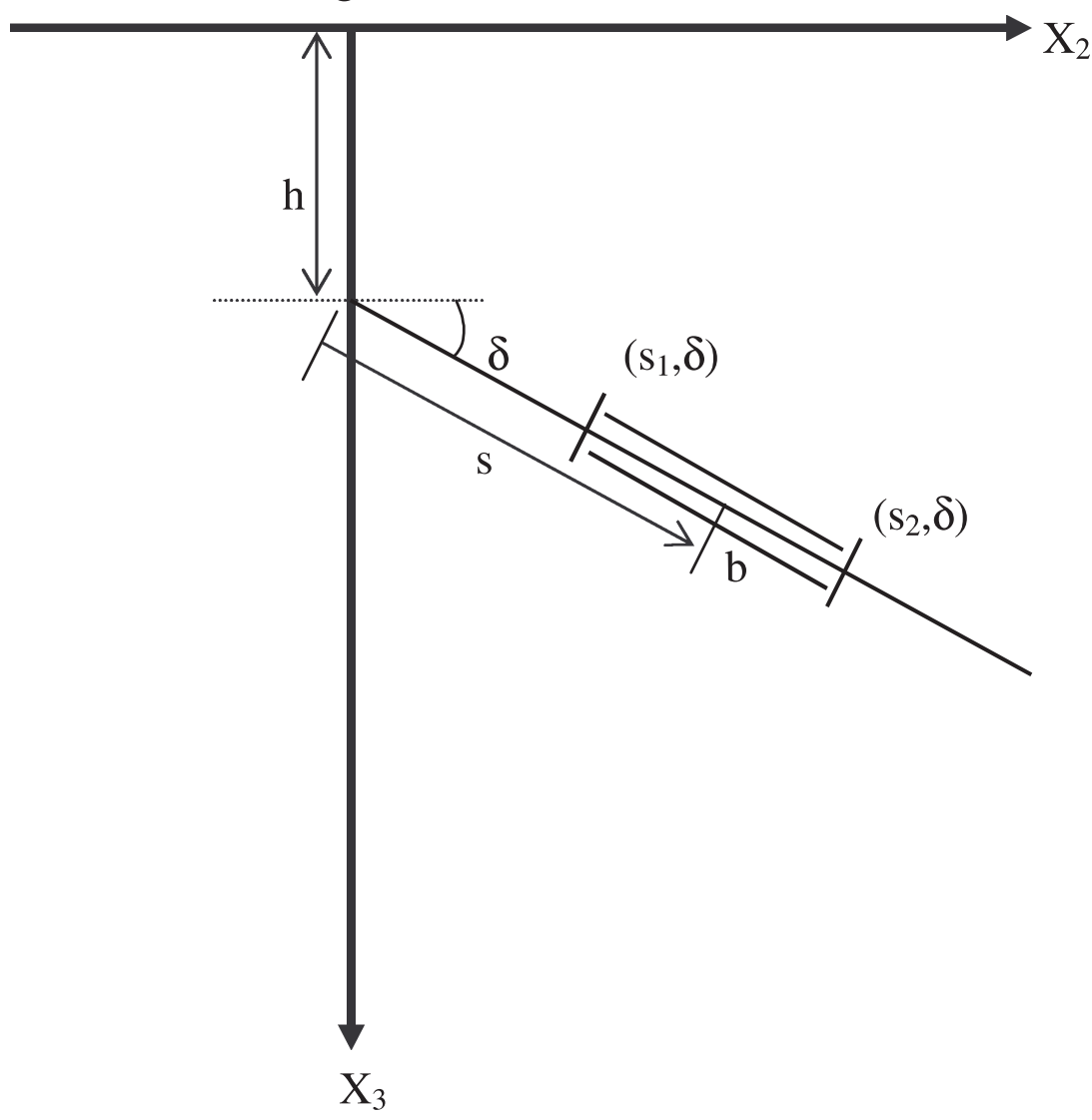

Figure 1. Geometry of the problem.

$p_{33}=\frac{\mu b}{2 \pi(1-\sigma)}\left\{\left(x_{2} \sin \delta+x_{3} \cos \delta-h \cos \delta\right)\right.$

$\left(\frac{1}{R_{1}^{2}}-\frac{1}{R_{2}^{2}}\right)-s \sin 2 \delta\left(\frac{1}{R_{1}^{2}}-\frac{1}{R_{2}^{2}}\right)$

$+2\left(x_{2} \sin \delta-x_{3} \cos \delta+h \cos \delta\right)$

$\left[\left(x_{3}-h-s \sin \delta\right)^{2} \frac{1}{R_{1}^{4}}-\left(x_{3}+h+s \sin \delta\right)^{2} \frac{1}{R_{2}^{4}}\right]$

$+4 x_{3}\left\{s \sin \delta\left(x_{2} \sin \delta+3 x_{3} \cos \delta+s \sin 2 \delta\right)\right.$

$-2 h \sin \delta\left(x_{2}-s \cos \delta\right)+3 h\left[\cos \delta\left(x_{3}+h\right)\right.$

$\left.\left.+\sin \delta\left(x_{2}+s \cos \delta\right)\right]\right\} \frac{1}{R_{2}^{4}}-16\left(x_{3}+h+s \sin \delta\right)^{2}$

$x_{3}\left\{s \sin \delta\left(x_{2} \sin \delta+x_{3} \cos \delta\right)+h\left[\left(x_{3}+h\right) \cos \delta\right.\right.$

$\left.\left.\left.+\left(x_{2}+s \cos \delta\right) \sin \delta\right]\right\} \frac{1}{R_{2}^{6}}\right\}\left.\right|_{0} ^{L}$.

Next, using the equations (3) and (5) into the relations between displacements and stresses (see Sokolnikoff 1956; pp: 265), we obtain

$$
\begin{aligned}
u_{2} & =\frac{b}{2 \pi}\left[\frac{(1-2 \sigma)}{2(1-\sigma)} \sin \delta \ln \frac{R_{1}}{R_{2}}\right. \\
& -\cos \delta \tan ^{-1}\left(\frac{x_{2}-s \cos \delta}{x_{3}-h-s \sin \delta}\right) \\
& +\cos \delta \tan ^{-1}\left(\frac{x_{2}-s \cos \delta}{x_{3}+h+s \sin \delta}\right) \\
& -\frac{1}{2(1-\sigma)}\left(x_{2} \sin \delta-x_{3} \cos \delta+h \cos \delta\right) \\
& \times\left(x_{2}-s \cos \delta\right)\left(\frac{1}{R_{1}^{2}}-\frac{1}{R_{2}^{2}}\right) \\
& -\frac{1}{(1-\sigma)} x_{3} \sin \delta(s \sin \delta+h) \frac{1}{R_{2}^{2}} \\
& +\frac{2}{\left(x_{3}+h+s \sin \delta\right)^{2}}\left[s \sin \delta\left(x_{3}+s \sin \delta\right)\right. \\
& \left\{\left(x_{3}+h+s \sin \delta\right)^{2} \sin \delta-\left(x_{3}+s \sin \delta\right)\right. \\
& \left.\left(x_{2}-s \cos \delta\right) \cos \delta\right\}-h\left\{\cos \delta\left(x_{2}-s \cos \delta\right)\right. \\
& {\left[\left(x_{3}+h+s \sin \delta\right)^{2}+x_{3} s \sin \delta\right] }
\end{aligned}
$$




$$
\begin{aligned}
& +\sin \delta\left(x_{3}+2 s \sin \delta+h\right)\left[s \cos \delta\left(x_{2}-s \cos \delta\right)\right. \\
& \left.\left.\left.-\left(x_{3}+s \sin \delta+h\right)^{2}\right]\right\}\right] \frac{1}{R_{2}^{2}} \\
& +\frac{2}{(1-\sigma)}\left(x_{2}-s \cos \delta\right) x_{3} \\
& {\left[s \sin \delta\left(x_{2} \sin \delta+x_{3} \cos \delta\right)+h\left\{\left(x_{3}+h\right) \cos \delta\right.\right.} \\
& \left.\left.\left.+\left(x_{2}+s \cos \delta\right) \sin \delta\right\}\right] \frac{1}{R_{2}^{4}}\right]\left.\right|_{0} ^{L}, \\
& u_{3}=\frac{b}{2 \pi}\left[-\frac{(1-2 \sigma)}{2(1-\sigma)} \cos \delta \ln \frac{R_{1}}{R_{2}}\right. \\
& -\sin \delta \tan ^{-1}\left(\frac{x_{3}+s \sin \delta+h}{x_{2}-s \cos \delta}\right) \\
& +\sin \delta \tan ^{-1}\left(\frac{x_{3}-s \sin \delta-h}{x_{2}-s \cos \delta}\right) \\
& +2(s \sin \delta+h)\left(x_{2} \sin \delta+x_{3} \cos \delta+h \cos \delta\right) \frac{1}{R_{2}^{2}} \\
& -\frac{1}{(1-\sigma)}\left[s \sin \delta\left(x_{2} \sin \delta+2 x_{3} \cos \delta\right)\right. \\
& \left.+h\left\{\left(2 x_{3}+h\right) \cos \delta+\left(x_{2}+s \cos \delta\right) \sin \delta\right\}\right] \frac{1}{R_{2}^{2}} \\
& -\frac{1}{2(1-\sigma)}\left(x_{2} \sin \delta-x_{3} \cos \delta+h \cos \delta\right) \\
& {\left[\left(x_{3}-h-s \sin \delta\right) \frac{1}{R_{1}^{2}}-\left(x_{3}+h+s \sin \delta\right) \frac{1}{R_{2}^{2}}\right]} \\
& +\frac{2}{(1-\sigma)}\left(x_{3}+h+s \sin \delta\right) x_{3} \\
& {\left[s \sin \delta\left(x_{2} \sin \delta+x_{3} \cos \delta\right)+h\left\{\left(x_{3}+h\right) \cos \delta\right.\right.} \\
& \left.\left.\left.+\left(x_{2}+s \cos \delta\right) \sin \delta\right\}\right] \frac{1}{R_{2}^{4}}\right]\left.\right|_{0} ^{L} .
\end{aligned}
$$

It is clear from the formulae (3)-(7) that the stresses and displacements depend on the depth of the upper edge of the fault $h$ and the dip angle $\delta$.

\section{Particular cases}

If we take $h=0$, then the problem reduces to that considered by Rani and Singh (1992b). In this case, putting $h=0$ into equations (3)-(7), we obtain the expressions of stresses and displacements given by
(11)-(15) of Rani and Singh (1992b) for the relevant problem. It can be verified that by putting both $x_{3}=0$ and $h=0$ into equations (6) and (7), one can obtain the same expressions of displacements as given by Freund and Barnett (1976) (cf. Rani and Singh (1992b) for corrections in their results).

\section{Numerical results and discussion}

We shall compute the stresses and the displacements numerically on the surface of the elastic halfspace containing a long dip-slip inclined fault of width $L$. We therefore take $s_{1}=0$ and $s_{2}=L$. We also assume that $\sigma=0.25$ and adopt the notations for non-dimensional quantities given in (13) of Rani and Singh (1992) alongwith $H=h / L$. When $\delta=90^{\circ}$, the fault is vertical. Figure 2 shows the variation of horizontal displacement $U_{2}$ with the distance from the fault, $Y$ for three positions of the fault, namely $H=0,1$ and 2 . We notice from this figure that the maximum value of $U_{2}$ is attained at $Y=0$. The value of $U_{2}$ decreases monotonically as $Y$ increases. We also notice that the maximum value of $U_{2}$ is equal to one at $Y=0$ on the surface, irrespective of the value of depth parameter $H$, while for all other values of $Y>0$, the value of $U_{2}$ depends on $H$. Figure 3 shows the variation of vertical displacement $U_{3}$ with the distance from the fault. We notice that the value of $U_{3}$ on the half-space is different for different value of $H$ for $Y>0$. Its value is maximum in magnitude at $Y=0$, irrespective of the position of the fault and it decreases as $Y$ increases and ultimately dies out as $Y$ approaches to infinity. Figure 4 shows the variation of $U_{2}$ with $\mathrm{Y}$, when fault is inclined at an angle $\delta=45^{\circ}$. We notice from this figure that $U_{2}$ is discontinuous at origin for inclined surface breaking fault $(H=0)$, while it is continuous for all values of $Y$ for inclined buried faults $(H=1$ and 2). Figure 5 shows the variation of $U_{2}$ with $Y$, when fault is horizontal i.e., when the angle $\delta=0^{0}$. We notice from this figure that the displacement $U_{2}$ is continuous for all values of $Y$ and is symmetrical about $Y=0.5$. Here, we would like to mention that the displacement $U_{2}$ could not be calculated at $Z=0$ for $H=0$. The reason being that in this case, since $\delta=0^{0}$, so physically the fault is along the surface of the elastic half-space, which is meaningless. Figures $6-8$ show the contour maps for stresses around a long vertical dip-slip fault whose upper edge is at $h=L$. We observed on comparing the contour maps for stresses with that of Rani and Singh (1992b) that these maps are influenced greatly by the depth parameter $H$. The contour maps for displacements are shown through figures 9 and 10 for $H=1$. These maps exhibit 


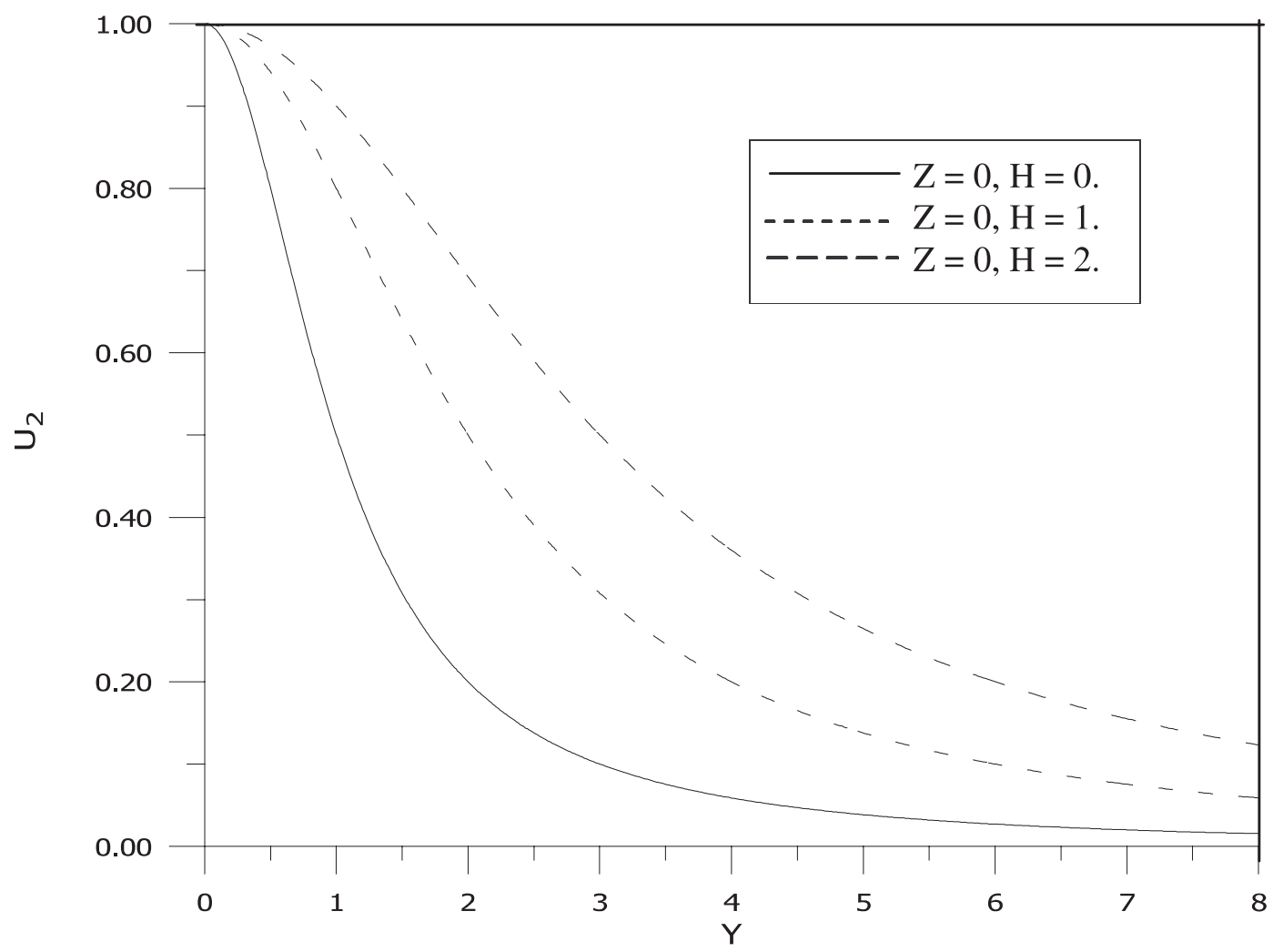

Figure 2. Variation of $U_{2}$ versus $Y$ at dip angle $\delta=90^{\circ}$.

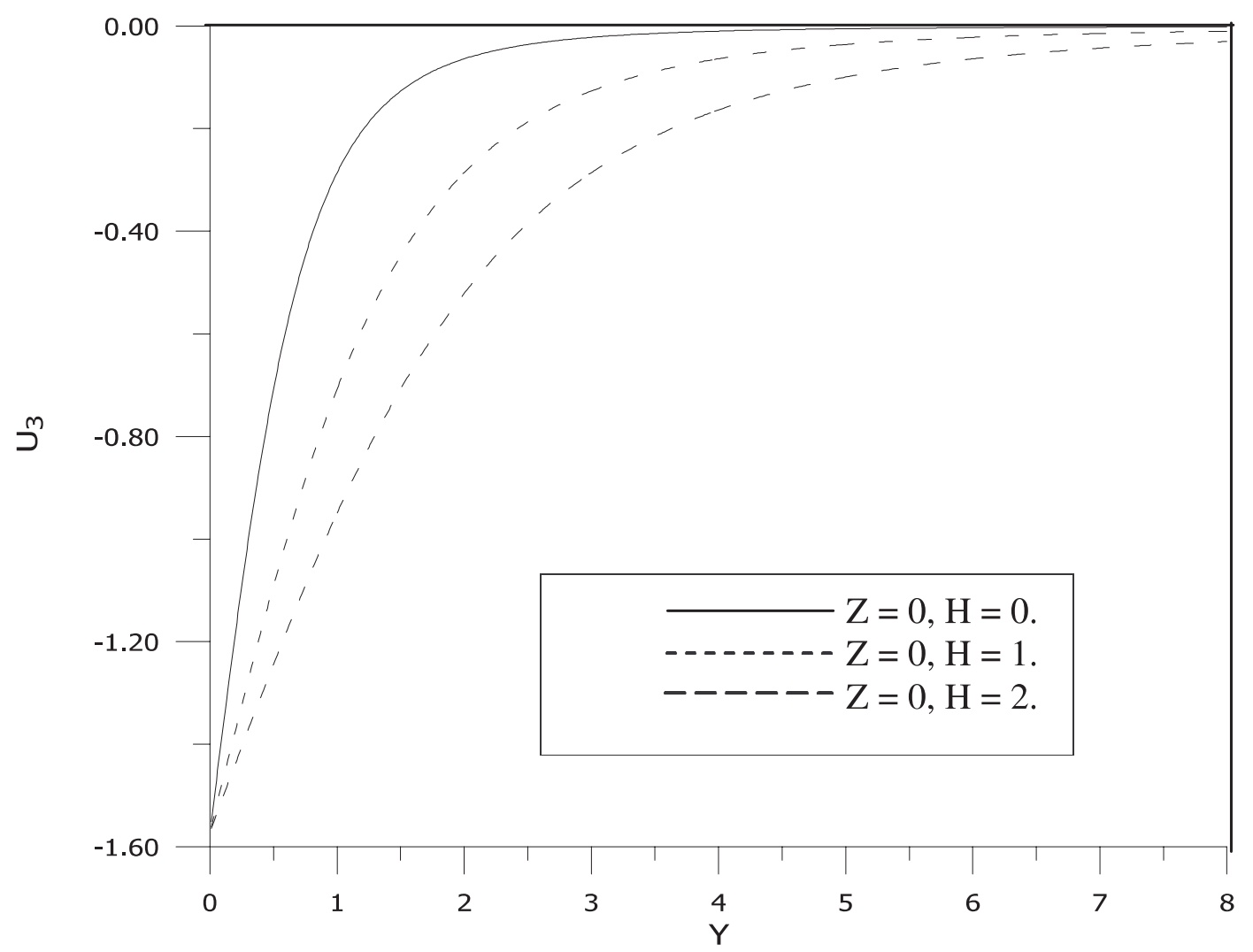

Figure 3. Variation of $U_{3}$ versus $Y$ at dip angle $\delta=90^{\circ}$. 


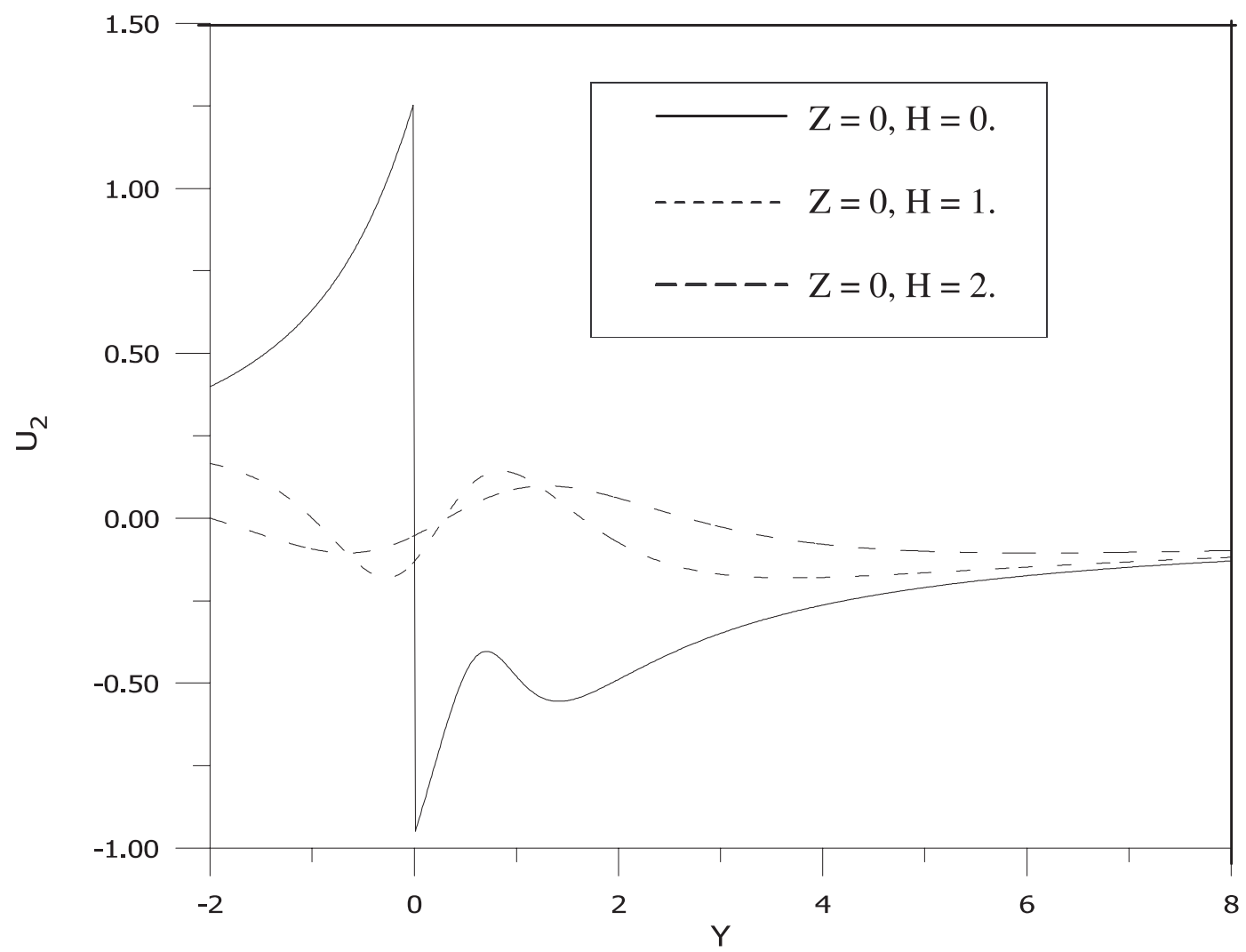

Figure 4. Variation of $U_{2}$ versus $Y$ at dip angle $\delta=45^{\circ}$.

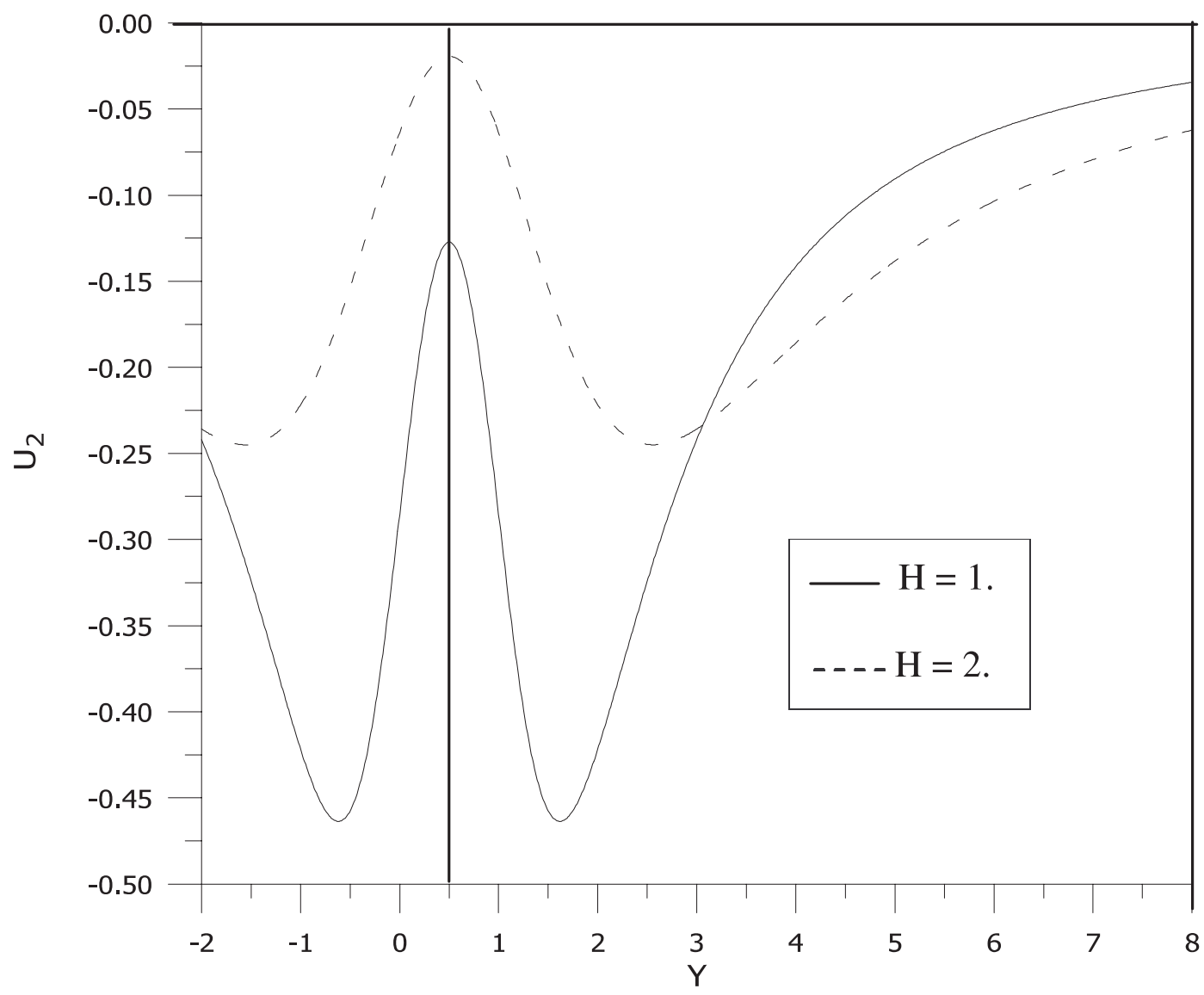

Figure 5. Variation of $U_{2}$ versus $Y$ at dip angle $\delta=0^{0}$. 

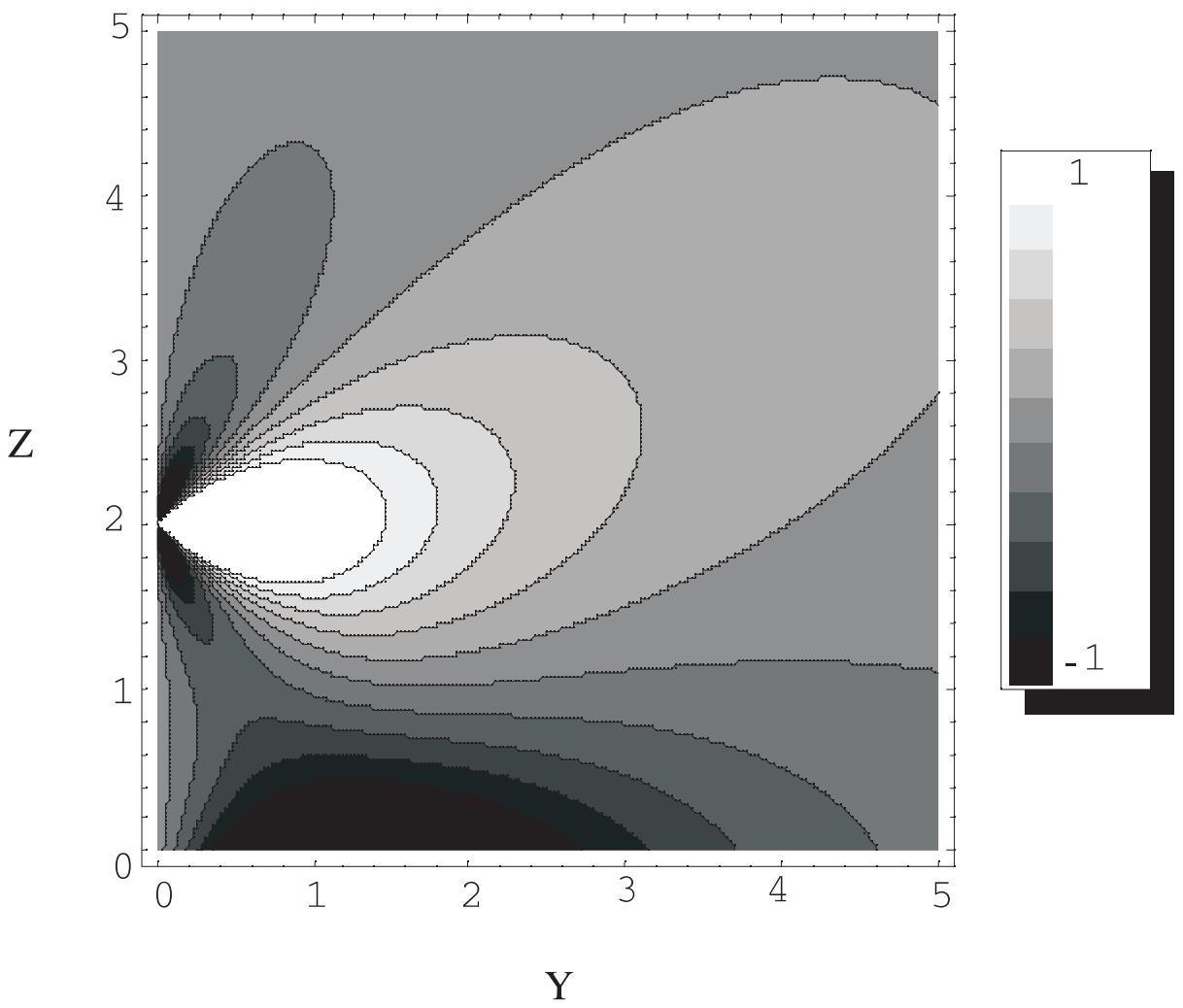

Figure 6. Contour map for $P_{22}$ when $\delta=90^{\circ}$ and $H=1$.

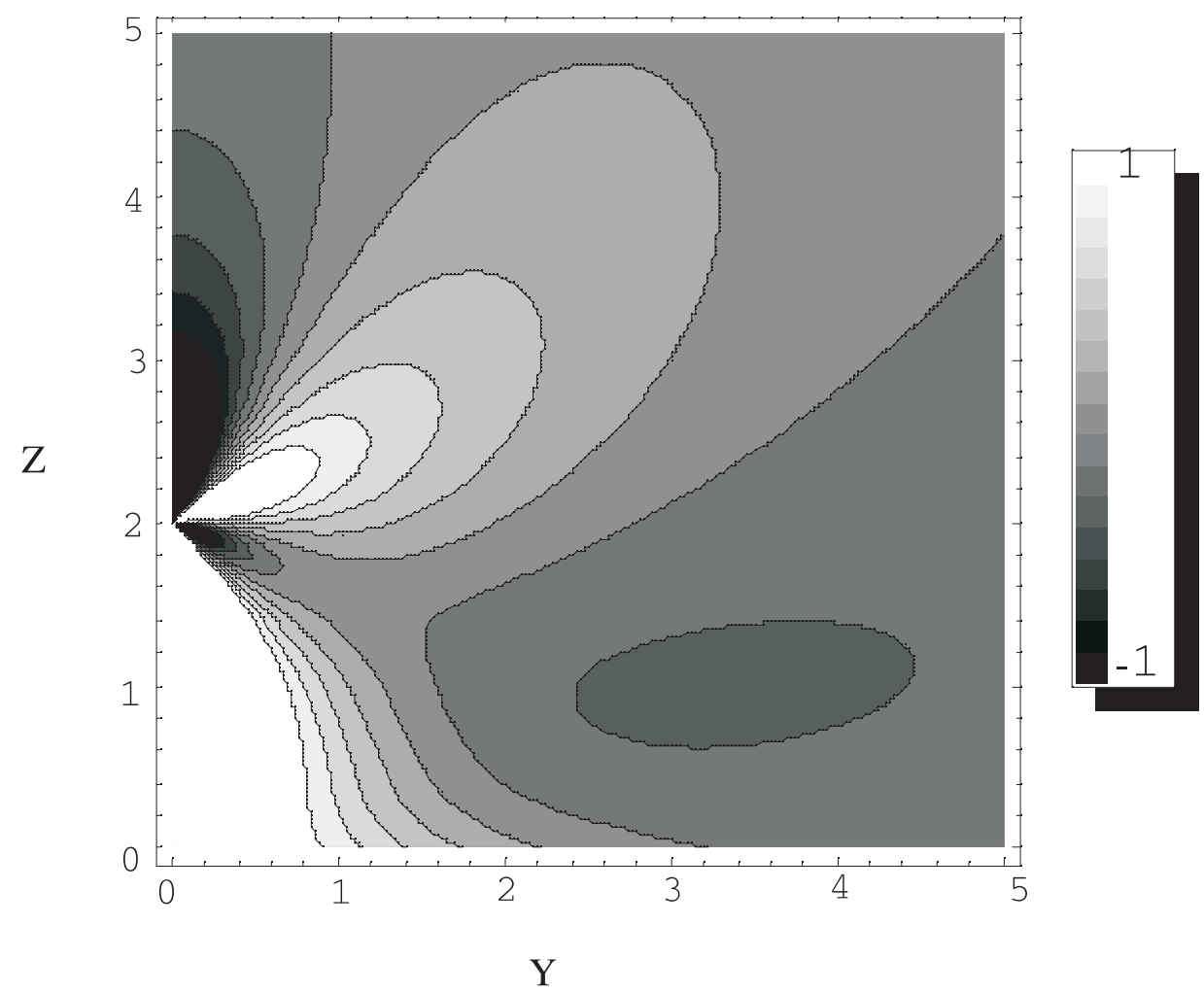

Figure 7. Contour map for $P_{23}$ when $\delta=90^{\circ}$ and $H=1$. 


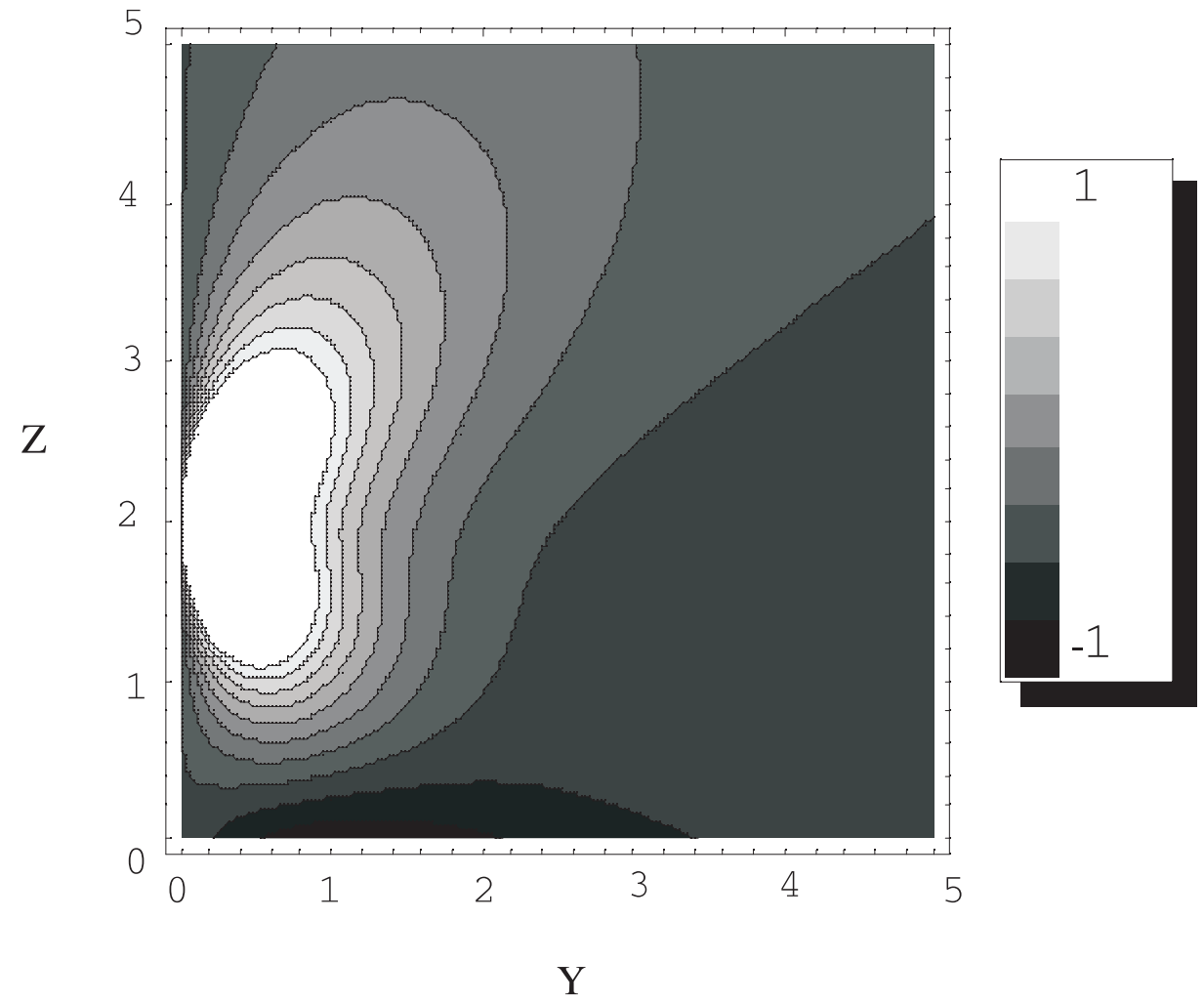

Figure 8. Contour map for $P_{33}$ when $\delta=90^{\circ}$ and $H=1$.

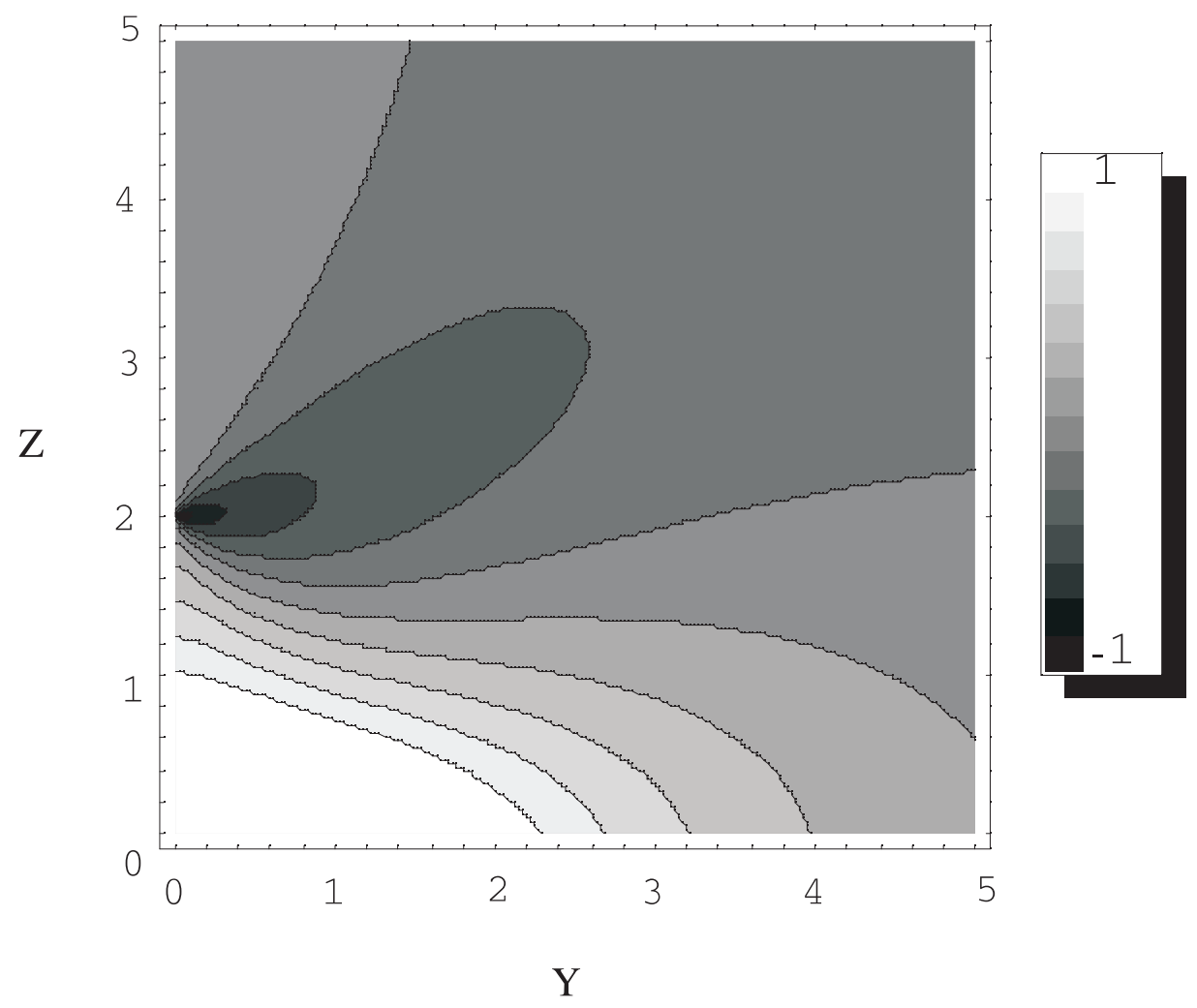

Figure 9. Contour map for $U_{2}$ when $\delta=90^{\circ}$ and $H=1$. 


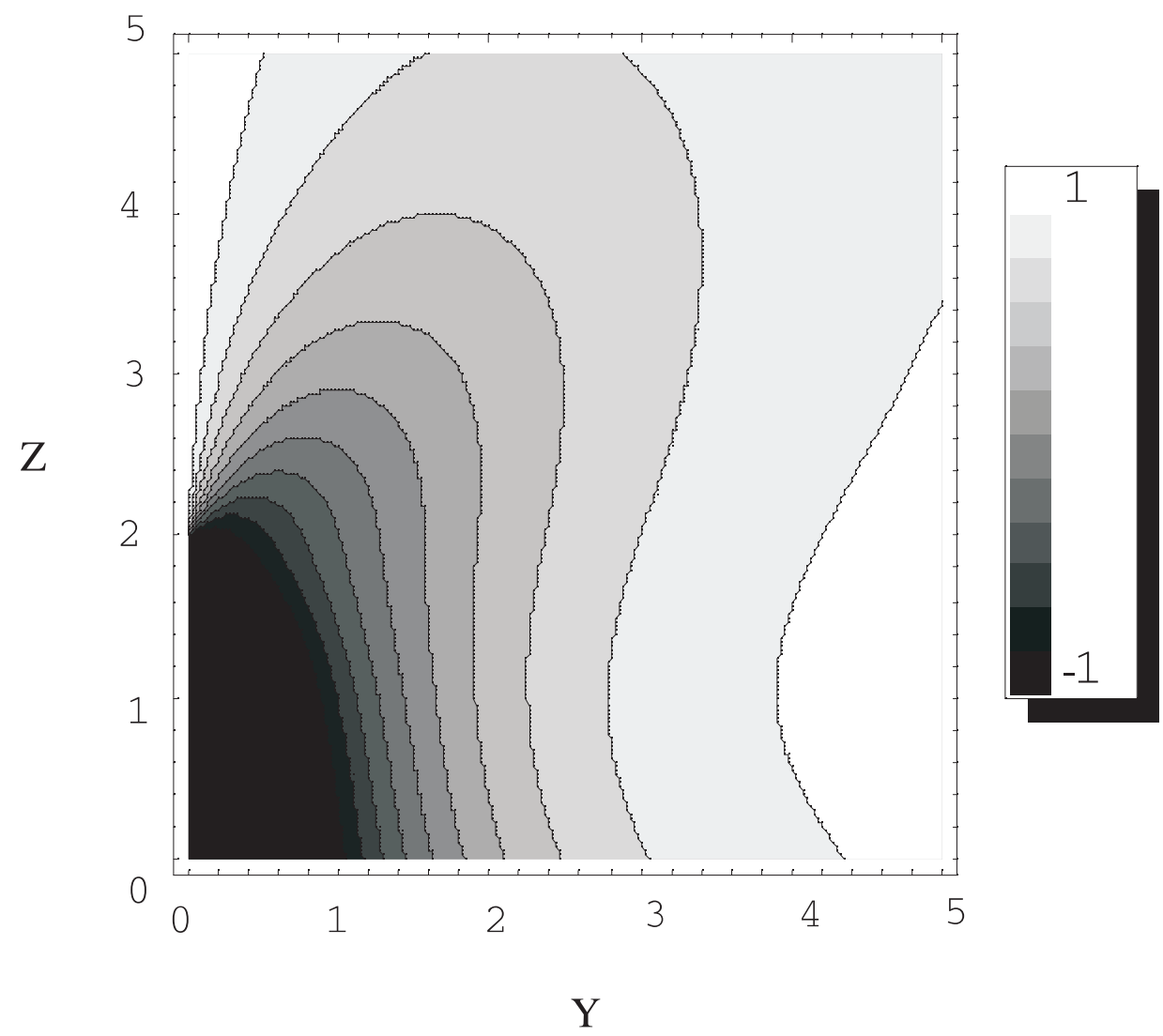

Figure 10. Contour map for $U_{3}$ when $\delta=90^{\circ}$ and $H=1$.

the variation of displacement field around the vertical dip-slip fault. In the legend of contour maps, symbol "1" stands for maximum value and "-1" stands for minimum value.

\section{Acknowledgements}

Authors are thankful to Prof. S J Singh and Prof. Harinder Singh for useful discussions and valuable suggestions in this work.

\section{References}

Cohen S C 1992 Postseismic deformation and stress diffusion due to visco-elasticity and comments on the modified Elsasser model; J. Geophys. Res. 97 15395-15403

Cohen S C 1996 Convenient formulas for determining dipslip fault parameters from geophysical observables; Bull. Seis. Soc. Am. 86 1642-1644

Freund L B and Barnett D M 1976 A two dimensional analysis of surface deformation due to dip-slip faulting; Bull. Seis. Soc. Am. 66 667-675

Maruyama T 1964 Statical elastic dislocations in an infinite and semi-infinite medium; Bull. Earthq. Res. Inst. 42 289-368

Maruyama T 1966 On two dimensional elastic dislocations in an infinite and semi-infinite medium; Bull. Earthq. Res. Inst. 44 811-871
Okada Y 1985 Surface deformation due to shear and tensile faults in a half-space; Bull. Seis. Soc. Am. 75 $1135-1154$

Okada Y 1992 Internal deformation due to shear and tensile faults in a half-space; Bull. Seis. Soc. Am. 82 $1018-1040$

Rani S, Singh S J and Garg N R 1991 Displacements and stresses at any point of a uniform half-space due to twodimensional buried sources; Phys. Earth. Planet Inter. 65 $276-282$

Rani S and Singh S J 1992a Static deformation of two welded half-spaces due to dip-slip faulting; Proc. Indian Acad. Sci.(Earth Planet. Sci.) 101 (3) 269-282

Rani S and Singh S J 1992b Static deformation of a uniform half-space due to a long dip-slip fault; Geophys. J. Int. $109469-476$

Rybicki K 1986 Dislocations and their geophysical applications. In: Continuum Theories in Solid Earth Physics, (ed) Tiesseyre R (Amsterdam: Elsevier) pp 18-186

Savage J C 1974 Dislocations in seismology. In: Dislocation theory: A treatise, (ed) F R N Nabarro (New York: Marcel Dekker)

Savage J C 1980 Dislocations in seismology. In: Dislocations in Solids, Vol. 3, Moving Dislocations, (ed) F R N Nabarro (Amsterdam: North-Holland) pp 251-339

Singh S J and Rani S 1993 Crustal deformation associated with two dimensional thrust faulting; J. Phys. Earth 41 87-101

Singh S J, Punia M and Rani S 1994 Crustal deformation due to non-uniform slip along a long fault; Geophys. J. Int. 118 411-427

Singh S J and Rani S 1996 2-D modeling of crustal deformation associated with strike-slip and dip-slip fault- 
ing in the Earth; Proc. Nat. Acad. Scs. (India) LXVI $187-215$

Singh S J, Punia M and Kumari G 1997 Deformation of a layered half-space due to a very long dip-slip fault; Proc. Indian Nat. Sci. Acad. 63A 225-240

Singh S J, Rani S and Garg N R 1992 Displacements and stresses in two welded half-spaces due to two-dimensional sources; Phys. Earth. Planet. Int. 70 90-101
Singh M and Singh S J 2000 Static deformation of a uniform half-space due to a very long tensile fault; ISET J. Earthq. Tech. 37(1-3) 27-38

Singh S J, Kumar A, Rani S and Singh M 2002 Deformation of a uniform half-space due to a long inclined tensile fault; Geophys. J. Int. 151957

Sokolnikoff I S 1956 Mathematical Theory of Elasticity (New York: McGraw-Hill)

MS received 25 July 2002; revised 21 January 2003 